\title{
Inequalities for Extreme Zeros of Some Classical Orthogonal and q-orthogonal Polynomials
}

\author{
K. Driver ${ }^{1 *}, \mathrm{~K}$. Jordaan ${ }^{2}$ \\ ${ }^{1}$ Department of Mathematics and Applied Mathematics, University of Cape Town 7701, RSA \\ 2 Department of Mathematics and Applied Mathematics, University of Pretoria, Pretoria, 0002, RSA
}

\begin{abstract}
Let $\left\{p_{n}\right\}_{n=0}^{\infty}$ be a sequence of orthogonal polynomials. We briefly review properties of $p_{n}$ that have been used to derive upper and lower bounds for the largest and smallest zero of $p_{n}$. Bounds for the extreme zeros of Laguerre, Jacobi and Gegenbauer polynomials that have been obtained using different approaches are numerically compared and new bounds for extreme zeros of q-Laguerre and little q-Jacobi polynomials are proved.
\end{abstract}

Keywords and phrases: Bounds for extreme zeros of orthogonal and q-orthogonal polynomials, common zeros of orthogonal polynomials, monotonicity, convexity, interlacing of zeros, separation of zeros, inequalities for zeros.

Mathematics Subject Classification: 33C45, 42C05

\section{Introduction}

If $\mu$ is a positive Borel measure, there exists a sequence of polynomials $\left\{p_{n}\right\}_{n=0}^{\infty}$, uniquely determined up to normalisation, that is orthogonal with respect to $\mu$. A sequence of orthogonal polynomials $\left\{p_{n}\right\}_{n=0}^{\infty}$ satisfies a three term recurrence relation of the form [46, Theorem 3.2.1]

$$
p_{n+1}(x)=\left(A_{n} x+B_{n}\right) p_{n}(x)-\lambda_{n} p_{n-1}(x),
$$

where $A_{n}, B_{n}$ and $\lambda_{n}$ are constants with $p_{-1}(x)=0, p_{0}(x)=1$ and $A_{n}>0, \lambda_{n}>0$ for each $n \in \mathbb{N}$. In the converse direction, Favard's Theorem proves that a polynomial sequence $\left\{p_{n}\right\}_{n=0}^{\infty}$ that satisfies a three-term recurrence relation of the form (1.1) with $A_{n}>0$ and $\lambda_{n}>0$ for each $n \in \mathbb{N}$ is orthogonal with respect to some positive Borel measure $\mu$. It is also well known that if $\left\{p_{n}\right\}_{n=0}^{\infty}$ is an orthogonal sequence then for each $n \in \mathbb{N}$, the $n$ zeros of $p_{n}$ are real and simple and lie in the convex hull of the support of $\mu$ [46, Theorem 3.3.1]. The properties and behavior of the zeros of orthogonal polynomials have attracted significant interest from both theoreticians and numerical analysts since the first results were proved by Markov [36] and Stieltjes [44] in the late 19th century. Reasons for this interest include the fact that zeros of orthogonal polynomials play an important role in Gauss quadrature formulas and interpolation problems and are also useful in proving classical inequalities. Electrostatic interpretations of the zeros of the classical orthogonal polynomials originally given by Stieltjes (cf. [46, Chapter 6]) and generalised by Ismail (cf. $[27,28]$ ) provide further reasons for interest in the properties of the zeros.

\footnotetext{
* Corresponding author. E-mail: kathy.driver@uct.ac.za
} 


\section{Properties of zeros of orthogonal polynomials}

An extensive discussion on the properties of the zeros of general orthogonal polynomials, as well as results specific to individual classical families, can be found in [46]. We note that if the measure of orthogonality $\mu$ is absolutely continuous, we write $d \mu(x)=w(x) d x$ where $w(x)$ is a weight function that is positive on the interval of orthogonality.

\subsection{Monotonicity of the zeros}

A central tool in proving inequalities satisfied by zeros of polynomials $p_{n}$ and $q_{n}, n \in \mathbb{N}$, where $\left\{p_{n}\right\}_{n=0}^{\infty}$ and $\left\{q_{n}\right\}_{n=0}^{\infty}$ are sequences that are orthogonal with respect to weight functions $w(x)$ and $W(x)$ respectively, is Markov's Theorem [36]. In the cases of classical orthogonal families such as Laguerre, Gegenbauer and Jacobi polynomials, the associated weight function is parameter-dependent and a corollary of Markov's Theorem shows that the zeros of polynomials within each orthogonal sequence vary monotonically with respect to the parameter(s) (cf. [46]). The application of Markov's Theorem to a specific orthogonal sequence $\left\{p_{n}\right\}_{n=0}^{\infty}$ requires the calculation of the derivative of the weight function and this is straightforward for many classical orthogonal families. However, it is more difficult and sometimes even impossible to apply Markov's Theorem to sequences that are orthogonal with respect to complicated weight functions and then alternative tools are required. Several techniques have been developed to derive monotonicity properties of zeros including the analysis of zeros of polynomial solutions of second-order ordinary linear differential equations (cf. [38] and [24, Chapter 7]) as well as the use of Wall-Wetzel's theorem on eigenvalues of Jacobi matrices in terms of chain sequences [49]. In [25,26] Ismail derived monotonicity results for the extreme zeros of polynomials involved in birth-and-death processes by using the coefficients of the three-term recurrence relation. Following Ismail's idea, the monotonicity of the extremal zeros of ultraspherical polynomials was investigated by Elbert and Siafarikas [15] while Erb and Tookos [16] applied a version of Ismail's theorem to prove corresponding monotonicity results for zeros of associated Jacobi, associated Gegenbauer and q-Meixner-Pollaczek polynomials. Ismail and Zhang [29] applied the Hellmann-Feynman Theorem while Ismail and Muldoon [30] used the tridiagonal matrices arising from the three term recurrence relation to study monotonicity properties of various special functions and orthogonal polynomials. Dimitrov and Rodrigues [11] invoked the classical Routh-Hurwitz stability criterion to obtain monotonicity results for the zeros of Jacobi polynomials.

\subsection{Spacing of the zeros}

Convexity properties of zeros are often useful in obtaining bounds for the distance between consecutive zeros. Sturm's comparison theorem can be used to show that the sequence of zeros of the solutions of second-order differential equations in normal form $y^{\prime \prime}+f(t) y=0$, is convex (concave) on an interval if $f(t)$ is increasing (decreasing) on that interval (cf. [45]). This monotonicity of the distance between consecutive zeros with respect to a parameter, or the order, following from Sturm's result has been studied by various authors for zeros of gamma, $q$-gamma, Bessel, cylindrical and Hermite functions and is discussed in detail in the survey paper [39].

The second order differential equations satisfied by polynomials such as Hermite, Laguerre, Gegenbauer and Jacobi are not in normal form and hence Sturm's theorem can not be applied directly but it is possible to apply a change of the variable to transform the differential equations into normal form. Sturm [45] and Hille [22] each use a simple transformation under which the zeros are invariant to prove the convexity of zeros of Bessel functions and Hermite polynomials respectively. The same transformation is used in [32] to find convexity properties and bounds on the distance between consecutive zeros of Laguerre, Gegenbauer and Jacobi polynomials. Szegö [46, Theorem 6.3.3] considers another change of variable which transforms the zeros and obtains information on the convexity of the transformed zeros of Gegenbauer polynomials while Deaño, Gil and Segura $[7,8]$ apply the same transformation to hypergeometric functions and study the convexity properties of their transformed zeros. The spacing of zeros of Laguerre, Gegenbauer and Jacobi polynomials, as the degree changes, is considered in [1]. A Sturm-type convexity theorem for 
difference equations is given in [19] and used to analyse the convexity of the zeros of discrete orthogonal polynomials such as Hahn and Meixner polynomials as well as q-Laguerre polynomials.

\subsection{Inequalities for the zeros}

Sturm's comparison theorem is used in [13] and [14] to obtain inequalities for the zeros of Gegenbauer and Jacobi polynomials respectively and similar classical methods are also described in [46]. Since the zeros of $p_{n}$ are real whenever $\left\{p_{n}\right\}_{n=0}^{\infty}$ is an orthogonal sequence, techniques developed to study the properties of zeros of polynomials in the Laguerre-Polya class are useful. Krasikov [34,35] as well as Dimitrov and Nikolov [10] use inequalities satisfied by polynomials with only real roots to derive bounds for the extreme zeros of Jacobi, Gegenbauer and Laguerre polynomials. The inequalities forming the basis of these derivations were conjectured by Foster and Krasikov [17], proved by Nikolov and Uluchev [41], and are refinements of Jensen inequalities for functions in the Laguerre-Polya class. Inequalities satisfied by the zeros of orthogonal and associated polynomials are derived by Ifantis and Siafarikas [23] and Siafarikas [42]. Limits for upper (lower) bounds of the largest (smallest) zeros for Gegenbauer and Hermite polynomials can be found in [2] while for Laguerre polynomials, monotonicity properties of their zeros are used by Dimitrov and Rafaeli [9] to establish analogous limits. Upper and lower bounds are obtained for each of the $n$ zeros in the cases of some classical families, with prominent attention being given to the "outside" bounds for the extreme zeros, namely, lower bounds for the smallest zero and upper bounds for the largest zero of polynomials in classical orthogonal families in [31] and [47]. Recent results include upper and lower bounds for all the zeros of Jacobi polynomials derived by Area, Dimitrov, Godoy and Rafaeli in [3].

\subsection{Interlacing and common zeros}

If $\left\{p_{n}\right\}_{n=0}^{\infty}$ is an orthogonal sequence, the zeros of $p_{n}$ interlace with the zeros of $p_{n-1}$ in the sense that each open interval with endpoints at successive zeros of $p_{n}$ contains exactly one zero of $p_{n-1}$. Stieltjes (cf. [46]), Theorem 3.3.3) extended this interlacing property by proving that if $m<n-1$, provided $p_{m}$ and $p_{n}$ have no common zeros, there exist $m$ open intervals with endpoints at successive zeros of $p_{n}$ each of which contains exactly one zero of $p_{m}$. Beardon (cf. [4], Theorem 5) provided additional insight into the Stieltjes interlacing process by proving that for every $m<n-1$, if $p_{m}$ and $p_{n}$ are co-prime, there exists a real polynomial $S_{n-m}$ of degree $n-m-1$ whose real simple zeros, together with those of $p_{m}$, interlace with the zeros of $p_{n}$. Iteration of the three term recurrence relation (1.1) leads to

$$
S_{n-m-1}(x) p_{n}(x)=S_{n-m}(x) p_{n-1}(x)-(-1)^{n-m-1} \lambda_{m} \ldots \lambda_{n-2} p_{m}(x),
$$

where $m<n-1, m, n \in \mathbb{N}$, and $\operatorname{deg} S_{m}=m-1$ for all $m \geq 2$. It should be noted that the polynomials $S_{n-m}$ are the dual polynomials introduced by de Boor and Saff in [6] where the following problems are solved: Given $n$ real distinct points and $k-1$ real distinct points, a) find conditions such that the monic polynomials $p_{n}$ and $q_{k-1}$ with zeros at the $n$ and $k-1$ given points respectively are members of the same sequence of orthogonal polynomials and b) when a) occurs, find all possible weight functions of orthogonality supported at $n$ points. Properties of the dual (or associated) polynomials $S_{n-m}$ are also analysed by Vinet and Zhedanov in [48].

Two obvious questions are whether it is necessary to assume that $p_{m}$ and $p_{n}$ are co-prime for $m<n-1$ in order to prove the interlacing results of Stieltjes and Beardon and whether there is numerical evidence of the occurrence of common zeros of polynomials within a classical orthogonal sequence. Given $m$ and $n, m<n-1$, the precise number of common zeros of two polynomials $p_{m}$ and $p_{n}$ belonging to a general orthogonal sequence $\left\{p_{n}\right\}_{n=0}^{\infty}$ is not known although there is an upper bound for the maximum possible number of common zeros, namely, min $[m, n-m-1]$, which was proved by Gibson in [18] and this upper bound is sharp. We recall Bourget's Hypothesis for Bessel functions (cf. [50]) which states that two Bessel functions $J_{n}$ and $J_{n+m}$ have no common zeros except at the origin for $m, n \in N$ and $m>1$. The first proof was given by Siegel in 1929 (cf. [43]) where he proved that Bourget's Hypothesis is true and that it follows 
as a simple corollary of his more general result that if $\nu$ is a rational number and $z$ is an algebraic number different from zero, then $J_{\nu}(z)$ is not an algebraic number. It turns out that the analogue of Bourget's Hypothesis does not hold in general for sequences of Gegenbauer, Laguerre or Jacobi polynomials since common zeros of polynomials of non-consecutive degree within these classical orthogonal sequences can be numerically found using Mathematica to compute the resultant of two polynomials $p_{n}$ and $p_{m}$ within each of these sequences. However, it can be proved (cf. [12]) that the largest or smallest zero of $p_{n}$ cannot also be a zero of $p_{m}$ for $m<n-1$ and that any two consecutive zeros of $p_{n}$ cannot both be zeros of $p_{m}$. These restrictions on the possible location of common zeros of $p_{m}$ and $p_{n}, m<n-1$ turn out to be useful for deducing good "inside" bounds for extremal zeros of orthogonal polynomials, namely upper (lower) bounds for the smallest (largest) zeros of $p_{n}$ in an orthogonal sequence $\left\{p_{n}\right\}_{n=0}^{\infty}$.

\subsection{Mixed three term recurrence relations and bounds for the extreme zeros}

The following result is proved in [12].

Theorem 2.1. [12] Let $\left\{p_{n}\right\}_{n=0}^{\infty}$ be a sequence of polynomials orthogonal on the (finite or infinite) interval $(c, d)$. Let $k, n \in \mathbb{N}$ be fixed with $k<n-1$ and suppose $g_{n-k}$ is a polynomial of degree $n-k-1$ that satisfies

$$
f(x) g_{n-k}(x)=G_{k}(x) p_{n-1}(x)+H(x) p_{n}(x)
$$

where $f(x) \neq 0$ for $x \in(c, d)$ and $H(x), G_{k}(x)$ are polynomials with $\operatorname{deg}\left(G_{k}\right)=k$. Then

(i) the $n-1$ real, simple zeros of $G_{k} g_{n-k}$ interlace with the zeros of $p_{n}$ if $g_{n-k}$ and $p_{n}$ are co-prime;

(ii) if $g_{n-k}$ and $p_{n}$ are not co-prime and have $r$ common zeros counting multiplicity, then

a) $r \leq \min \{k, n-k-1\}$;

b) these $r$ common zeros are simple zeros of $G_{k}$;

c) no two successive zeros of $p_{n}$, nor its largest or smallest zero, can also be zeros of $g_{n-k}$;

d) the $n-2 r-1$ zeros of $G_{k} g_{n-k}$, none of which is also a zero of $p_{n}$, together with the $r$ common zeros of $g_{n-k}$ and $p_{n}$, interlace with the $n-r$ remaining (non-common) zeros of $p_{n}$.

Equation (2.2) and the conditions of Theorem 2.1 hold for each $k, n \in \mathbb{N}$ fixed, $k<n-1$, with $g_{n-k}=$ $p_{n-k-1}$ as can be seen (cf. [4, Theorem 4]) by iterating the three term recurrence relation satisfied by an orthogonal sequence $\left\{p_{n}\right\}_{n=0}^{\infty}$. Mixed recurrence relations typically satisfied by classical orthogonal polynomials corresponding to different values of the parameter(s) provide functional equations with the structure given in (2.2) linking polynomials from distinct sequences within the same classical orthogonal family.

The following corollary of Theorem 2.1 provides a surprisingly simple tool for deriving good bounds for the extreme zeros of orthogonal polynomials.

Corollary 2.2. [12] Suppose the conditions of Theorem 2.1 hold. Then the largest (smallest) zero of $G_{k}$ is a strict lower (upper) bound for the largest zero of $p_{n}$.

Mixed three term recurrence relations of the type given in (2.2) that involve the largest possible parameter difference between the polynomials, or alternatively, no parameter difference but the largest possible difference in the degree, while still preserving interlacing properties for their zeros, are particularly useful for finding sharp bounds.

\section{Examples}

We numerically compare bounds for the extreme zeros of Laguerre, Gegenbauer and Jacobi polynomials that have been obtained by different authors using a variety of methods. We will see that the comparative sharpness of the bounds varies with the values of the parameters and with $n$. We denote the zeros of the polynomial $p_{n}$ of degree $n$ by $w_{1}<\cdots<w_{n}$. 


\subsection{Bounds for the extreme zeros of Gegenbauer polynomials}

The sequence of Gegenbauer (or ultraspherical) polynomials $\left\{C_{n}^{\lambda}\right\}_{n=0}^{\infty}$ is the one-parameter classical family obtained from the sequence of Jacobi polynomials $\left\{P_{n}^{\alpha, \beta}\right\}_{n=0}^{\infty}$ with $\alpha=\beta=\lambda-\frac{1}{2}$. The sequence is orthogonal on $[-1,1]$ with respect to the weight function $\left(1-x^{2}\right)^{\lambda-\frac{1}{2}}$ for $\lambda>-\frac{1}{2}, n \in \mathbb{N}$. The zeros of $C_{n}^{\lambda}$ lie in the open interval $(-1,1)$ and are symmetric about the origin with a simple zero at the origin when $n$ is odd. It was shown in [12] that

$$
\begin{aligned}
& w_{n}^{2}>1-\frac{(2 \lambda+1)(2 \lambda+3)}{(n-1)(n+2 \lambda+1)+(2 \lambda+1)(2 \lambda+3)}, \\
& w_{n}>1-\frac{(2 \lambda+1)(2 \lambda+5)}{(4 n-4)(n+2 \lambda+1)+(2 \lambda+1)(2 \lambda+5)} .
\end{aligned}
$$

In table 1 we compare (3.1) and (3.2) to the bound given by Szego (cf. [46, eqn. (6.2.13)])

$$
w_{n}^{2} \geq 1-\frac{2 \lambda+1}{n+2 \lambda}
$$

TABLE 1. Comparison of lower bounds for the largest zero of $C_{n}^{\lambda}(x)$ for different values of $n$ and $\lambda$.

\begin{tabular}{|c|c|c|c|c|}
\hline Value of $n$ and $\lambda$ & $n=31, \lambda=-0.49$ & $n=4, \lambda=-0.4956$ & $n=51, \lambda=30.9$ & $n=6, \lambda=60.49$ \\
\hline Largest zero of $C_{n}^{\lambda}$ & 0.999978 & 0.999267 & 0.897416 & 0.289296 \\
\hline Bound (3.2) given in [12] & 0.999978 & 0.999267 & 0.844369 & 0.142782 \\
\hline Bound (3.1) given in [12] & 0.999978 & 0.999266 & 0.763561 & 0.201482 \\
\hline Bound (3.3) given in [46] & 0.999667 & 0.998537 & 0.66578 & 0.198435 \\
\hline
\end{tabular}

\subsection{Bounds for the extreme zeros of Jacobi polynomials}

The sequence of Jacobi polynomials $\left\{P_{n}^{\alpha, \beta}\right\}_{n=0}^{\infty}$ is orthogonal on the interval $(-1,1)$ with respect to the weight function $(1-x)^{\alpha}(1+x)^{\beta}$ for $\alpha, \beta>-1$. It was shown in [12] that for fixed $n \in \mathbb{N}$ and $\alpha, \beta>-1$,

$$
w_{n}>1-\frac{2(\alpha+1)(\alpha+3)}{(2 n-2)(n+\alpha+\beta+2)+(\alpha+3)(\alpha+\beta+2)}=1-O\left(\frac{1}{n^{2}}\right)
$$

and

$$
w_{1}<-1+\frac{2(\beta+1)(\beta+3)}{(2 n-2)(n+\alpha+\beta+2)+(\beta+3)(\alpha+\beta+2)}=-1+O\left(\frac{1}{n^{2}}\right),
$$

improving the bounds given by Szegö in [46, eqns. (6.2.11) and (6.2.12)], namely

$$
\begin{aligned}
& w_{n}>1-\frac{2(\alpha+1)}{2 n+\alpha+\beta}=1-O\left(\frac{1}{n}\right) \\
& w_{n}>1-\frac{\alpha+1}{n+\alpha}=1-O\left(\frac{1}{n}\right) \text { for } \beta \geq \alpha .
\end{aligned}
$$


It is also clear from table 2 that (3.4) is significantly sharper than the recent bound obtained in [3, Cor. $3]$

$$
w_{n}>\frac{K_{n, n}(\beta)-K_{n, 1}(\alpha)}{K_{n, n}(\beta)+K_{n, 1}(\alpha)}
$$

with

$$
\begin{aligned}
& K_{n, n}(a)=(\sqrt{n+a+1}-\sqrt{n})^{2}+3(\sqrt{n+a+1}-\sqrt{n})^{\frac{4}{3}}\left((\sqrt{n+a+1}+\sqrt{n})^{2}-(\sqrt{n+a+1}-\sqrt{n})^{2}\right)^{-\frac{1}{3}} \\
& K_{n, 1}(a)=(\sqrt{n+a+1}+\sqrt{n})^{2}-3(\sqrt{n+a+1}+\sqrt{n})^{\frac{4}{3}}\left((\sqrt{n+a+1}+\sqrt{n})^{2}-(\sqrt{n+a+1}-\sqrt{n})^{2}\right)^{-\frac{1}{3}}+2,
\end{aligned}
$$

even for the case where this bound performs the best, i.e. when the difference between the parameters $\alpha$ and $\beta$ is large.

TABLE 2. Comparison of lower bounds for the largest zero of $P_{n}^{\alpha, \beta}(x)$ for different values of $n, \alpha$ and $\beta$.

\begin{tabular}{|c|c|c|c|c|c|c|}
\hline Value of $n$ & $n=4$ & $n=16$ & $n=12$ & $n=19$ & $n=4$ & $n=4$ \\
\hline Value of $\alpha$ & $\alpha=-0.9$ & $\alpha=-0.9$ & $\alpha=30.9$ & $\alpha=30.9$ & $\alpha=0.9$ & $\alpha=-0.5$ \\
\hline Value of $\beta$ & $\beta=-0.8$ & $\beta=-0.8$ & $\beta=-0.8$ & $b=32.8$ & $\beta=132.8$ & $\beta=3000$ \\
\hline Largest zero of $P_{n}^{\alpha, \beta}$ & 0.984119 & 0.999143 & 0.141417 & 0.71025 & 0.989891 & 0.999903 \\
\hline Bound (3.4) given in [12] & 0.984109 & 0.999142 & -0.0507338 & 0.590098 & 0.989162 & 0.999902 \\
\hline Bound (3.6) given in [46] & 0.968254 & 0.993399 & -0.179298 & 0.372665 & 0.973183 & 0.999667 \\
\hline Bound (3.7) given in [46] & 0.967742 & 0.993377 & NA & 0.360721 & 0.612245 & 0.857143 \\
\hline Bound (3.8) given in [3] & -0.983346 & -0.996718 & -0.998007 & -0.567437 & -0.233866 & 0.9925 \\
\hline
\end{tabular}

In table 3 we compare (3.5) to numerical values of the smallest zero as well as the upper bound for the smallest zero

$$
w_{1}<\frac{K_{n, 1}(\beta)-K_{1,1}(\alpha)}{K_{n, 1}(\beta)+K_{1,1}(\alpha)}
$$

obtained in [3].

TABle 3. Comparison of upper bounds for the smallest zero of $P_{n}^{\alpha, \beta}(x)$ for different values of $n, \alpha$ and $\beta$.

\begin{tabular}{|c|c|c|c|c|c|c|}
\hline Value of $n$ & $n=4$ & $n=16$ & $n=12$ & $n=19$ & $n=4$ & $n=4$ \\
\hline Value of $\alpha$ & $\alpha=-0.9$ & $\alpha=-0.9$ & $\alpha=30.9$ & $\alpha=30.9$ & $\alpha=0.9$ & $\alpha=-0.5$ \\
\hline Value of $\beta$ & $\beta=-0.8$ & $\beta=-0.8$ & $\beta=-0.8$ & $b=32.8$ & $\beta=132.8$ & $\beta=3000$ \\
\hline Smallest zero of $P_{n}^{\alpha, \beta}$ & -0.998208 & -0.998208 & -0.998457 & -0.682434 & 0.84914 & 0.994291 \\
\hline Bound (3.5) given in [12] & -0.966742 & -0.998203 & -0.999155 & -0.551942 & 0.886203 & 0.995674 \\
\hline Bound (3.9) given in [3] & 0.993421 & 0.999895 & 0.445731 & 0.831911 & 0.982603 & 0.999814 \\
\hline
\end{tabular}




\subsection{Upper bounds for the smallest zeros of Laguerre polynomials}

The sequence of Laguerre polynomials $\left\{L_{n}^{\alpha}\right\}_{n=0}^{\infty}$ is orthogonal on the interval $(0, \infty)$ with respect to the weight function $x^{\alpha} e^{-x}$ for $\alpha>-1$. Upper bounds for the smallest zero $w_{1}$ of the Laguerre polynomial $L_{n}^{\alpha}, \alpha>-1$, namely

$$
w_{1}<\frac{(\alpha+1)(\alpha+2)}{n+\alpha+1}
$$

and

$$
w_{1}<\frac{(\alpha+1)(\alpha+3)}{2 n+\alpha+1}
$$

were obtained by Hahn (cf. [21]) and Szegö (cf. [46, eqn. (6.31.12)]) respectively. Gupta and Muldoon recovered these bounds in [20, eqns. (2.9) and (2.10)]. In [12, eqn. (4)] the upper bound for the smallest zero of $L_{n}^{\alpha}$ is

$$
\frac{(\alpha+2)_{2}(3 n+2 \alpha+2)-\sqrt{(\alpha+2)_{2}\left(9(\alpha+2)_{2}+2(2 \alpha+5)\left(\alpha^{2}+5 \alpha+10\right)(n-1)+\left(5 \alpha^{2}+25 \alpha+38\right)(n-1)^{2}\right)}}{2(n+\alpha+1)_{2}} .
$$

A sharper upper bound was derived by Gupta and Muldoon in [20, eqn. (2.11)], namely

$$
w_{1}<\frac{(\alpha+1)(\alpha+2)(\alpha+4)(2 n+\alpha+1)}{(\alpha+1)^{2}(\alpha+2)+n(5 \alpha+11)(n+\alpha+1)} .
$$

TABLE 4. Comparison of upper bounds for the smallest zero of $L_{n}^{\alpha}(x)$ for different values of $n$ and $\alpha$.

\begin{tabular}{|c|c|c|c|c|}
\hline Values of $\alpha$ and $n$ & $\alpha=340.56, n=13$ & $\alpha=65.3, n=21$ & $\alpha=-0.9, n=101$ & $\alpha=-0.9, n=4$ \\
\hline Smallest zero of $L_{n}^{\alpha}$ & 251.815653 & 27.67770854 & 0.00103831 & 0.0259078 \\
\hline Bound (3.12) given in [12] & 275.856 & 32.6035 & 0.00103832 & 0.0259079 \\
\hline Bound (3.13) given in [20] & 309.594 & 36.6163 & 0.00103833 & 0.0259082 \\
\hline Bound (3.11) given in [20] & 319.258 & 41.8125 & 0.00103909 & 0.0259259 \\
\hline Bound (3.10) given in [20] & 330 & 51.111 & 0.00108803 & 0.268293 \\
\hline
\end{tabular}

Remark. As observed in [20], it is evident from (3.10), (3.11) and (3.13) that the upper bounds for the smallest zero will be sharpest for $\alpha$ close to -1 .

\subsection{Lower bounds for the largest zeros of Laguerre polynomials}

The lower bound for the largest zero $w_{n}$ of $L_{n}^{\alpha}(x), \alpha>-1$

$$
w_{n}>4 n+\alpha-16 \sqrt{2 n}
$$

is given by Bottema [5] while

$$
w_{n}>3 n-4
$$

in [40] is due to Neumann and

$$
w_{n}>2 n+\alpha-1
$$


was obtained by Szegö (cf. [46, eqn. (6.2.14)]). In [12], the lower bound for the largest zero of $L_{n}^{\alpha}$

$$
w_{n}>2 n+\alpha-2+\sqrt{n^{2}-2 n+\alpha n+2}
$$

is given. Although (3.17) is an improvement on previous bounds for most values of $n$ and $\alpha$, the bound (3.15) due to Neumann, which does not depend on $\alpha$, compares favourably when $\alpha$ is small while Bottema's bound (3.14) is better when $n$ is large as illustrated in table 5 .

TABLE 5. Comparison of lower bounds for the largest zero of $L_{n}^{\alpha}(x)$ for different values of $n$ and $\alpha$.

\begin{tabular}{|c|c|c|c|c|}
\hline Values of $\alpha$ and $n$ & $\alpha=340.56, n=13$ & $\alpha=65.3, n=21$ & $\alpha=-0.9, n=101$ & $\alpha=-0.9, n=5$ \\
\hline Largest zero of $L_{n}^{\alpha}$ & 469.74362252 & 172.7701885 & 591.362 & 11.1263 \\
\hline Bound (3.17) given in [12] & 429.612 & 146.616 & 298.654 & 10.7606 \\
\hline Bound (3.16) given in [46] & 365.56 & 106.3 & 200.1 & 8.1 \\
\hline Bound (3.14) given in [5] & 310.976 & 45.6081 & 175.69 & -31.4964 \\
\hline Bound (3.15) given in [40] & 35 & 59 & 299 & 11 \\
\hline
\end{tabular}

\section{New bounds for the extreme zeros of q-orthogonal polynomials}

Iterations of the three term recurrence relation satisfied by $q$-orthogonal polynomials can be used to derive bounds for the largest and smallest zeros of q-orthogonal polynomials.

\section{1. q-Laguerre}

The $q$-Laguerre polynomials defined by (cf. [37])

$$
L_{n}^{\alpha}(x ; q)=\frac{\left(q^{\alpha+1} ; q\right)_{n}}{(q ; q)_{n}} \sum_{k=0}^{n} \frac{\left(q^{-n} ; q\right)_{k} q^{2}(1-q)^{k}\left(q^{\alpha+n+1} x\right)^{k}}{\left(q^{\alpha+1} ; q\right)_{k}(q ; q)_{k}}, q \in(0,1)
$$

where

$$
\begin{aligned}
& (a ; q)_{k}=(1-a)(1-a q) \ldots\left(1-a q^{k-1}\right), k=1,2,3 \ldots \\
& (a ; q)_{0}=1
\end{aligned}
$$

are orthogonal on $(0, \infty)$ for $\alpha>-1$ and satisfy the three term recurrence relation

$$
\begin{aligned}
q\left(1-q^{n+\alpha-1}\right) L_{n-2}^{\alpha} & =A_{n} L_{n-1}^{\alpha}-\left(1-q^{n}\right) L_{n} \\
A_{n} & =q\left(1-q^{n-1}\right)+\left(1-q^{n+\alpha}\right)-q^{2 n+\alpha-1} x(1-q) .
\end{aligned}
$$

The zero of the coefficient of $L_{n-1}$ in (4.1) yields the bound

$$
w_{1}<\frac{q^{1-\alpha-2 n}\left(q^{n+\alpha}+q^{n}-q-1\right)}{(q-1)}<w_{n} .
$$

Iterating (4.1) we obtain

$$
\begin{aligned}
q^{2}\left(1-q^{n+\alpha-1}\right)\left(1-q^{n+\alpha-2}\right) L_{n-3}^{\alpha} & \left.=\left[B_{n+1} B_{n-1}-q\left(1-q^{n-1}\right)\left(1-q^{n+\alpha-1}\right)\right] L_{n-1}^{\alpha}-\left(1-q^{n}\right) B_{n} L_{n}^{\alpha}\right] \\
B_{n} & =\left(1-q^{n}\right)+q\left(1-q^{n+\alpha-1}\right)-(1-q) q^{2 n+\alpha-1} x
\end{aligned}
$$


and the zeros of the quadratic polynomial coefficient of $L_{n-1}^{\alpha}$ in (4.3) yield the bounds

$$
\begin{aligned}
& w_{1}<\frac{(q-1) q^{2 n+\alpha+1}(q+1)\left(q^{n}+q^{n+\alpha}-q^{2}-1\right)+C_{n}}{2(q-1) q^{2 n+\alpha^{2}}} \\
< & \frac{(q-1) q^{2 n+\alpha+1}(q+1)\left(q^{n}+q^{n+\alpha}-q^{2}-1\right)-C_{n}}{2(q-1) q^{2 n+\alpha^{2}}}<w_{n}
\end{aligned}
$$

for the extreme zeros of $L_{n}^{\alpha}(x ; q), \alpha>-1,0<q<1$ where

$C_{n}=(q-1) q^{2 n+\alpha+1}$

$$
\sqrt{1-q^{2}-q^{4}+q^{6}+q^{2 n}+2\left(q+q^{5}-q^{n}-q^{4+n}-q^{1+2 n}\right)+q^{2+2 n}+(q-1)^{2} q^{2(n+\alpha)}+2 q^{n+\alpha}\left(q^{n}+q^{2+n}-1-q^{4}\right)} .
$$

Replacing $n$ by $n-2$ in [37, (4.14)] and using (4.1) to eliminate the term in $L_{n-2}^{\alpha}$ we have

$$
x L_{n-2}^{\alpha+1}=\frac{1-q^{n}}{q^{n+\alpha}(q-1)} L_{n}^{\alpha}-\frac{q^{2 n+\alpha-1}}{q^{n+\alpha}}\left(x-\frac{1-q^{n+\alpha}}{q^{2 n+\alpha-1}(1-q)}\right) L_{n-1}^{\alpha}
$$

and obtain the bound

$$
w_{1}<\frac{1-q^{n+\alpha}}{q^{2 n+\alpha-1}(1-q)}<w_{n}
$$

for the extreme zeros of $L_{n}^{\alpha}(x ; q), \alpha>-1,0<q<1$ from Corollary 2.2. Lastly, replacing $n$ by $n-1$ in [37, (4.13)] we obtain

$$
\frac{\left(1-q^{n+\alpha-1}\right)}{(1-q)} L_{n-1}^{\alpha-1}=\frac{\left(1-q^{n}\right)}{q^{n}(1-q)} L_{n}^{\alpha}+\left(q^{n+\alpha-1} x-\frac{1-q^{n}}{q^{n}(1-q)}\right) L_{n-1}^{\alpha}
$$

which yields another bound

$$
w_{1}<\frac{q^{1-2 n-\alpha}\left(q^{n}-1\right)}{q-1}<w_{n}
$$

for the extreme zeros of $L_{n}^{\alpha}(\mathrm{x} ; \mathrm{q}), \alpha>-1,0<q<1$.

In table 6 below we compare these new bounds to the actual values of the extreme zeros and the upper bound for the smallest zero

$$
w_{1}<\frac{\left(1-q^{\alpha+1}\right)\left(1-q^{\alpha+2}\right)(1+q)}{q^{\alpha+1}(1-q)\left(1+2 q-q^{n+\alpha+2}-q^{n}-q^{\alpha+2}\right)}
$$

of $L_{n}^{\alpha}(x ; q), \alpha>-1,0<q<1$ derived by Gupta and Muldoon in [20]. Although (4.8) is the best available upper bound for the smallest zero, the bounds following from Corollary 2.2 are significantly better lower bounds for the largest zero.

\subsection{Little q-Jacobi}

Little $q$-Jacobi polynomials may be defined by

$$
p_{n}(x ; a, b ; q)=\sum_{k=0}^{n} \frac{\left(q^{-n} ; q\right)_{k}\left(a b q^{n+1} ; q\right)_{k}}{(a q ; q)_{k}} \frac{(x q)^{k}}{(q ; q)_{k}}
$$

and are orthogonal on $(0, \infty)$ when $0<a q<1,0<b q<1$. These polynomials satisfy the three term recurrence relation (cf. [33, (14.12.3)])

$$
D_{n} p_{n-2}(x, a, b, q)=\left(E_{n}+D_{n}-x\right) p_{n-1}(x ; a, b ; q)-E_{n} p_{n}(x ; a, b ; q)
$$

where

$$
\begin{aligned}
D_{n} & =\frac{\left(a q^{n-1}\left(1-q^{n-1}\right)\left(1-b q^{n-1}\right)\right.}{\left(1-a b q^{2 n-2}\right)\left(1-a b q^{2 n-1}\right)} \text { and } \\
E_{n} & =\frac{q^{n-1}\left(1-a q^{n}\right)\left(1-a b q^{n}\right)}{\left(1-a b q^{2 n-1}\right)\left(1-a b q^{2 n}\right)}
\end{aligned}
$$


TABle 6. Comparison of bounds for the extreme zeros of $L_{n}^{\alpha}(x ; q)$ when $q=0.46$ for different values of $n$ and $\alpha$.

\begin{tabular}{|c|c|c|c|c|}
\hline Values of $\alpha$ and $n$ & $\alpha=2.13, n=10$ & $\alpha=65.3, n=20$ & $\alpha=-0.9, n=3$ & $\alpha=-0.9, n=100$ \\
\hline Largest zero of $L_{n}^{\alpha}$ & $3.80799 \times 10^{7}$ & $4.25881 \times 10^{35}$ & 54.0614 & $1.83064^{67}$ \\
\hline Bound (4.2) & $3.6119 \times 10^{7}$ & $4.0394 \times 10^{35}$ & 52.1582 & $1.73633^{67}$ \\
\hline Bound (4.4) & $5.68318 \times 10^{6}$ & $6.35827 \times 10^{34}$ & 5.87909 & $2.73309 \times 10^{66}$ \\
\hline Bound (4.5) & $3.80755 \times 10^{7}$ & $4.25831 \times 10^{35}$ & 54.0612 & $1.83043 \times 10^{67}$ \\
\hline Bound (4.6) & $3.64952 \times 10^{6}$ & $2.76671 \times 10^{35}$ & 35.9475 & $1.83043 \times 10^{67}$, \\
\hline Bound (4.7) & $3.64454 \times 10^{6}$ & $2.76671 \times 10^{35}$ & 40.3483 & $1.18927 \times 10^{67}$ \\
\hline Smallest zero of $L_{n}^{\alpha}$ & 12.3281 & $2.75067 \times 10^{22}$ & 0.0909758 & 0.0823258 \\
\hline Bound (4.8) in $[20]$ & 14.309 & $3.21969 \times 10^{22}$ & 0.0925009 & 0.0839114 \\
\hline
\end{tabular}

and it follows from Corollary 2.2 that the extreme zeros of $p_{n}(x ; a, b ; q)$ satisfy the inequality

$$
w_{1}<D_{n}+E_{n}<w_{n}
$$

when $0<a q<1$ and $0<b q<1$. Replacing $n$ by $n-1$ in (4.9) and substituting (4.9) into the resulting equation, we obtain

$$
\begin{aligned}
D_{n} D_{n-1} p_{n-3}(x ; a, b, q)= & \left(\left(D_{n-1}+F_{n-1}-x\right)\left(D_{n}+E_{n}-x\right)-D_{n} F_{n-1}\right) p_{n-1}(x ; a, b, q) \\
& -E_{n}\left(D_{n-1}+F_{n-1}-x\right) p_{n}(x ; a, b ; q)
\end{aligned}
$$

and this yields the bounds for the extreme zeros of $p_{n}(x ; a, b ; q)$ for $0<a q<1$ and $0<b q<1$

$$
\begin{aligned}
w_{1} & <F_{n}-\sqrt{F_{n}^{2}-\left(D_{n}+E_{n}\right)\left(D_{n-1}+E_{n-1}\right)+D_{n} E_{n-1}} \\
& <F_{n}+\sqrt{F_{n}^{2}-\left(D_{n}+E_{n}\right)\left(D_{n-1}+E_{n-1}\right)+D_{n} E_{n-1}}<w_{n}
\end{aligned}
$$

where

$$
F_{n}=\frac{D_{n-1}+E_{n-1}+D_{n}+E_{n}}{2} .
$$

In table 7 we compare these new bounds for the extreme zeros of $p_{n}(x, a, b, q)$ when $0<a q, b q<1$ to numerically computed values of the extreme zeros for certain values of the parameters observing that they cannot readily be compared to the bounds obtained for the smallest zero of $p_{n}(x(1-q) ; a, b ; q)$ when $0<a q<1, b<1$ in $[20]$.

TABLE 7. Comparison of bounds for the extreme zeros of $p_{n}(x ; a, b ; q)$ when $q=0.4$ and $n=3$ for different values of $a$ and $b$.

\begin{tabular}{|c|c|c|c|c|}
\hline Values of $a$ and $b$ & $a=0.2, b=0.3$ & $a=2.1, b=2.4$ & $a=2.1, b=0.4$ & $a=0.1, b=2.4$ \\
\hline Smallest zero of $p_{n}$ & 0.141326 & 0.0202896 & 0.0179588 & 0.151676 \\
\hline Largest zero of $p_{n}$ & 0.999997 & 0.9999 & 0.997032 & 1 \\
\hline Bound (4.10) & 0.183126 & 0.311491 & 0.404969 & 0.165425 \\
\hline Bound (4.11) & 0.147875 & 0.136213 & 0.268757 & 0.151742 \\
\hline Bound (4.12) & 0.4636180 & 0.41614 & 0.901398 & 0.401463 \\
\hline
\end{tabular}


Acknowledgements. Research by both authors is supported by the National Research Foundation of South Africa.

\section{References}

[1] S. Ahmed, A. Laforgia, M. E. Muldoon. On the spacing of the zeros of some classical orthogonal polynomials. J. London Math. Soc., second series 25 (1982), 246-252.

[2] I. Area, D.K. Dimitrov, E. Godoy, A. Ronveaux. Zeros of Gegenbauer and Hermite polynomials and connection coefficients. Math. Comp., 73 (2004), 1937-1951.

[3] I. Area, D.K. Dimitrov, E. Godoy, F.R. Rafaeli. Inequalities for zeros of Jacobi polynomials via Obrechkoff's theorem. Math. Comp., 81 (2012), 991-1004.

[4] A.F. Beardon. The theorems of Stieltjes and Favard. Lect. Notes Math., 11(1) (2011), 247-262.

[5] O. Bottema. Die Nulstellen gewisser durch Rekursionsformeln definierter Polynome. Proc. Amsterdam, 34 (5)(1931), 681.

[6] C. de Boor, EB Saff. Finite sequences of orthogonal polynomials connected by a Jacobi matrix. Linear Algebra Appl., 75 (1986), 43-55.

[7] A. Deaño, A. Gil, J. Segura. New inequalities from classical Sturm theorems. J. Approx. Theory, 131 (2004), $208-243$.

[8] A. Deaño, J. Segura. LG transformations and global inequalities for real zeros of Gauss hypergeometric functions. J. Approx. Theory, 48 (2007), 92-110.

[9] D.K. Dimitrov, F.R. Rafaeli. Monotonicity of zeros of Laguerre polynomials. J. Comput. Appl. Math.. 223 (2009), 699-702.

[10] D.K. Dimitrov, G.P. Nikolov. Sharp bounds for the extreme zeros of classical orthogonal polynomials. J. Approx. Theory, 162 (2010), 1793-1804.

[11] D.K. Dimitrov, R.O. Rodrigues. On the behaviour of zeros of Jacobi and Gegenbauer polynomials. J. Approx. Theory., 116 (2002), 224-239.

[12] K. Driver, K. Jordaan. Bounds for extreme zeros of some classical orthogonal polynomials. J. Approx. Theory., 164 (2012), 1200-1204.

[13] Á. Elbert, A. Laforgia. Upper bounds for the zeros of ultraspherical polynomials. J. Approx. Theory., 61 (1990), 88-97.

[14] Á. Elbert, A. Laforgia, L.G. Rodonó. On the zeros of Jacobi polynomials. Acta Math. Hungar., 64 (4) (1994), 351-359

[15] Á. Elbert, P.D. Siafarikas. Monotonicity properties of the zeros of ultraspherical polynomials. J. Approx. Theory., 97 (1999) 31-39.

[16] W. Erb, F. Tookós. Monotonicity of extremal zeros of orthogonal polynomials and applications. Appl. Math. Comput., 217 (2011), 4771-4780.

[17] W.H. Foster, I. Krasikov. Inequalities for real-root polynomials and entire functions. Adv. Appl. Math., 29 (2002), $102-114$.

[18] P.C. Gibson. Common zeros of two polynomials in an orthogonal sequence. J. Approx. Theory, 105 (2000), $129-132$.

[19] J. Gishe, F. Tookós. On the Sturm comparison and convexity theorem for difference and q-difference equations. Acta Scientiarum Mathematicarum. In press.

[20] D.E. Gupta, M.E. Muldoon. Inequalities for the smallest zeros of Laguerre polynomials and their q-analogues. Journal of Inequalities in Pure and Applied Mathematics, 8 (2007), Issue 1, Article 24, 7 pp.

[21] W. Hahn. Bericht über die Nullstellen der Laguerrschen und der Hermiteschen Polynome. Jahresbericht der Deutschen Mathematiker-Vereinigung, 44 (1933), 215-236.

[22] E. Hille. Über die Nulstellen der Hermiteschen Polynome. Jahresbericht der Deutschen Mathematiker-Vereinigung, 44 (1933), 162-165.

[23] E.K. Ifantis, P.D. Siafarikas. Differential inequalities on the greatest zero of Laguerre and ultraspherical polynomials in Actas del VI Simposium on Polinomios Orthogonales Y Aplicaciones, Gijon (1999) 187-197.

[24] M.E.H. Ismail. Classical and Quantum Orthogonal Polynomials in One Variable, Encyclopedia of Mathematics and its Applications, Cambridge: Cambridge University Press, 98 (2005).

[25] M.E.H. Ismail. The variation of zeros of certain orthogonal polynomials. Advances in Appl. Math., 8 (1987), $111-118$.

[26] M.E.H. Ismail. Monotonicity of zeros of orthogonal polynomials. Invited address in "q-Series and Partitions", edited by D. Stanton, IMA Volumes in Mathematics and its Applications, Vol. 18, Springer-Verlag, New York, 1989, 177-190.

[27] M.E.H. Ismail. An electrostatic model for zeros of general orthogonal polynomials. Pacific J. Math. 193 (2000), $355-369$.

[28] M.E.H. Ismail. More on elctrostatic models for zeros of orthogonal polynomials. J. Nonlinear Functional Analysis and Optimization, 21 (200, 43-55.

[29] M.E.H. Ismail, R Zhang, On the Hellmann-Feynman theorem and the variation of zeros of certain special functions. Adv. Appl. Math., 9 (1988), 439-446.

[30] M.E.H. Ismail, M.E. Muldoon, A discrete approach to monotonicity of zeros of orthogonal polynomials. Transactions Amer. Math. Soc., 323 (1991), 65-78.

[31] M.E.H. Ismail, X. Li. Bounds on the extreme zeros of orthogonal polynomials. Proc. Amer. Math. Soc., 115 (1992) 131-140.

[32] K. Jordaan, F. Tookós. Convexity of the zeros of some orthogonal polynomials and related functions. J. Comp. Anal. Appl., 233 (2009), 762-767.

[33] R. Koekoek, P.A. Lesky, R.F. Swarttouw. Hypergeometric orthogonal polynomials and their q-analogue. Springer Monographs in Mathematics, Springer Verlag, Berlin (2010). 
[34] I. Krasikov. Bounds for zeros of the Laguerre polynomials. J. Approx. Theory., 121 (2003), $287-291$.

[35] I. Krasikov. On zeros of polynomials and allied functions satisfying second order differential equations. East J. Approx., 9 (2003), 41-65.

[36] A. Markov. Sur les racines de certaines équations (seconde note). Math. Ann., 27 (1886) 177-182.

[37] D.S. Moak. The q-Analogue of the Laguerre Polynomials. J. Math. Anal. Appl., 81 (1981), $20-47$.

[38] M.E. Muldoon. Properties of zeros of orthogonal polynomials and related functions. J. Comput. Appl. Math., 48 (1993) $167-186$.

[39] M.E. Muldoon. Convexity properties of special functions and their zeros. Milovanovic, G. V. (ed.), Recent progress in inequalities. Dedicated to Prof. Dragoslav S. Mitrinovic. Dordrecht: Kluwer Academic Publishers. Math. Appl., Dordr. 430 1998, 309-323 Publishers, Inc., Boston, 1991.

[40] E.R. Neumann. Zur Theorie der Laguerreschen Polynome. Jahresber. d. S.M.V., 30 (1921) 15.

[41] G.P. Nikolov, R. Uluchev. Inequalities for real-root polynomials. Proof of a conjecture of Foster and Krasikov. in: D.K. Dimitrov, G.P. Nikolov, R. Uluchev (eds.), Approximation Theory: A volume dedicated to B. Bojanov. Marin Drinov Academic Publishing House, Sofia, 2004, pp. 201-216.

[42] P.D. Siafarikas. Inequalities for the zeros of the associated ultraspherical polynomials. Math. Inequal. Applic., 2(2), (1999) 233-241.

[43] C.L. Siegel. Über einige Anwendungen diophantischer Approximationen. Abh. Preuss. Akad. Wiss., 1, (1929), 1-70.

[44] T.J. Stieltjes. Sur les racines de l équation $X_{n}=0$. Acta Math., 9 (1886) 385-400.

[45] C. Sturm. Memoire sur les équations différentielles du second ordre. J. Math. Pures Appl., 1 (1836) $106-186$.

[46] G. Szegö. Orthogonal Polynomials. American Mathematical Society Colloquium Publications, Volume XXIII, Providence, RI, fourth edition, 1975.

[47] E. van Doorn. Representations and bounds for zeros of orthogonal polynomials and eigenvalues of sign-symmetric tri-diagonal matrices. J. Approx. Theory., 51 (1987) 254-266.

[48] L. Vinet, A. Zhedanov. A characterization of classical and semiclassical orthogonal polynomials from their dual polynomials. J. Comput. Appl. Math. 172 (2004), 41-48.

[49] H.S. Wall, M. Wetzel. Quadratic forms and convergence regions for continued fractions. Duke Math. J., 11 (1944), 983-1000.

[50] G.N. Watson. A Treatise on the Theory of Bessel Functions 2nd ed., (Cambridge University Press, Cambridge 1966). 